\title{
GEAR SCALE ESTIMATION FOR SYNTHETIC SPEED PULSE GENERATION
}

\author{
John-Olof Nilsson`, Isaac Skog, Alessio De Angelis, Claudia Aquilanti, and Peter Händel \\ Signal Processing Lab \\ ACCESS Linneaus Centre \\ KTH Royal Institute of Technology \\ Stockholm, Sweden \\ Email: ^jnil02@kth.se
}

\begin{abstract}
In a motorized vehicle a number of easily measurable signals with frequency components related to the rotational speed of the engine can be found, e.g., vibrations, electrical system voltage level, and ambient sound. These signals could potentially be used to estimate the speed and related states of the vehicle. Unfortunately, such estimates would typically require the relations (scale factors) between the frequency components and the speed for different gears to be known. Consequently, in this article we look at the problem of estimating these gear scale factors from training data consisting only of speed measurements and measurements of the signal in question. The estimation problem is formulated as a maximum likelihood estimation problem and heuristics is used to find initial values for a numerical evaluation of the estimator. Finally, a measurement campaign is conducted and the functionality of the estimation method is verified on real data.
\end{abstract}

Index Terms - In-car navigation, gear ratios, speed pulse, maximum likelihood estimation

\section{INTRODUCTION}

There are a number of measurable signals in a motorized vehicle with frequency components having linear relations to the rotational speed of the vehicle engine. For example vibrations induced by the running engine will be felt in the chassis [1]; Imperfections in the rectification of the generator output results in an AC component related to the rotation of the generator in the voltage level of the electrical system [2]; Even cylinder firing rate can to some extent be picked up in the ambient sound in the vehicle [3]. These signals could potentially be easily accessible sources of information for estimating the speed and related states of the vehicle. As a result this could limit the number of connections and sensors and allow for less restrictive choice of sensor types and sensor placements in a vehicle. Also these signals might provide external access to information about speed and related vehicle states. Especially, this could give a synthetic speedpulse signal to third-party dashboard navigation systems or to standalone navigation system implemented on smartphones or PDAs with built in GPS-receivers and inertial sensors [4]. However, to estimate the speed two additional pieces of information would be required: First, the relation between the speed and frequency component of interest for each gear; Second, knowledge about the engaged gear. Vice versa, given an estimate of the speed and the relation between the speed and frequency, these signals could be used to detect the engaged gear. ${ }^{1}$ The gear shifts and consequently the engaged gear can be detected with inertial sensors and an estimate of the speed can be acquired from a GPS receiver [5, 4]. Hence, in the absence of a GPS-fix, e.g. during a GPS outages, these signals could in combination with inertial sensor give estimates of the vehicle velocity and thereby improve the robustness and availability of the navigation system. Even without inertial sensors especially for short GPS outages we can get some information about the motion by assume that the engaged gear is not changed or by trying to use some pattern recognition to detect gear shifts. Together with for example mapmatching this could to some extent increase the availability of the navigation system. Also in general these signals will provide additional information to a navigation system improving overall performance. However, for any of this to be possible the unknown relation between the speed and frequency components of interest needs to be known.

For each gear the relation between the speed of the vehicle and the rotational speed of the engine is linear making the relation between speed and frequency component linear. In this article we suggest a batch processing method to estimate these linear relations (scale factors) based on readily available training data consisting of simultaneous measurements of speed and the signal in question. No information about engaged gears is assumed. However, the total number of (forward) gears and an approximate value of the ratios between the scale factors of subsequent gears are assumed known (see subsection 3.2 for comments about this). In this article only scale factors of forward gears (traveling in the forward direction) are considered, although the problem can be expanded to also include the scale factor of the reverse gear, mutatis mutandis. Measurement models are formulated and the scale factor estimation is posed as a maximum-likelihood (ML) estimation problem. The resulting estimator is non-linear, has high dimensionality, and contains several nuisance parameters. To overcome this, heuristic methods are used to fix some of the nuisance parameters and to find initial values for a numerical evaluation of the estimator. Finally, a measurement campaign with a car is conducted in which speed from GPS doppler measurements and electrical system voltage level are recorded. With the help of reference values the estimation method is shown to perform well on the recorded data.

\footnotetext{
${ }^{1}$ Throughout the article only mechanical transmissions of noncontinuously variable type are considered.
} 


\section{MEASUREMENT MODELS}

A frequency component of interest $\tilde{s}_{k}$ is assumed tracked over time in the signal of interest and given together with speed measurement $\tilde{v}_{k}$ for time instants $k$. The measurements $\tilde{s}_{k}$ and $\tilde{v}_{k}$ are assumed to be measures of the true quantities $s_{k}$ and $v_{k}$ via the relations

$$
\begin{aligned}
& \tilde{s}_{k}=s_{k}+w_{k}^{s}, \\
& \tilde{v}_{k}=v_{k}+w_{k}^{v},
\end{aligned}
$$

where $w_{k}^{s} \sim \mathcal{N}\left(0, \sigma_{s}^{2}\right)$ and $w_{k}^{v} \sim \mathcal{N}\left(0, \sigma_{v}^{2}\right)$ are independent white measurement noise components.

The vehicle is assumed to be traveling in forward direction for all time instants $k$. This assumption is needed since speed measurements do not distinguish between forward and backward motion. If speed or auxiliary training data that allow for such distinction is available this assumption can be relaxed. The relation between $s_{k}$ and $v_{k}$ will be dependent on the engaged gear $i$ and the state of the clutch (engaged or disengaged). The gearbox is assumed to have $M$ forward gears. If the clutch is engaged

$$
v_{k}=\theta_{i} s_{k} \quad \text { for some } i,
$$

where $\theta_{i}>0$ are the scale factors of the linear relations for the forward gears $i \in\{1,2, \ldots, M\}$. Together with (1) and (2) this gives

$$
\begin{aligned}
\tilde{v}_{k} & =\theta_{i} \tilde{s}_{k}-\theta_{i} w_{k}^{s}+w_{k}^{v} \\
& =\theta_{i} \tilde{s}_{k}+w_{k}\left(\theta_{i}\right) \text { for some } i,
\end{aligned}
$$

where $w\left(\theta_{i}\right)_{k} \sim \mathcal{N}\left(0, \theta_{i}^{2} \sigma_{s}^{2}+\sigma_{v}^{2}\right)$ is the combined effect of the measurement noise components $w_{k}^{s}$ and $w_{k}^{v}$. If the clutch is disengaged the relation between $v_{k}$ and $s_{k}$ and hence also between $\tilde{v}_{k}$ and $\tilde{s}_{k}$ is undetermined. Further, without loss of generality, the gear scale factors are assumed ordered. The combined constraints on the scale factors are then

$$
\theta_{i}<\theta_{j} \quad \forall i<j, \theta_{1}>0
$$

\section{SCALE FACTOR ESTIMATION}

If the engaged gear $i$ and the clutch state were given for each time instants $k$, estimating $\theta_{i}$ would be trivial. Unfortunately, the information about the engaged gear and the clutch state is typically not easily automatically accessible. Therefore, we would like to do without. The information given for a single measurement is quantified by (1)-(5). For multiple measurements there is clearly more information available from correlations in time in the gearbox and clutch states as well as in the velocity and frequency measurement, i.e., momentum consideration and driving behavior limit the speed at which the quantities of interest change. However, this information is difficult to quantify. Therefore it is ignored and $\tilde{v}_{k}$ and $\tilde{s}_{k}$ measurements for different $k$ are assumed independent.

From (4) and the notion of the undetermined relation when the clutch is disengaged, the probability density function (pdf) of $\tilde{v}_{k}$ conditioned on $\tilde{s}_{k}$ and parameterized by $\boldsymbol{\theta}=\left[\theta_{1}, \ldots, \theta_{M}\right]$ is

$$
p\left(\tilde{v}_{k} \mid \tilde{s}_{k} ; \boldsymbol{\theta}\right)=\sum_{i=1}^{M} \alpha_{i} \frac{1}{\sqrt{2 \pi\left(\theta_{i}^{2} \sigma_{s}^{2}+\sigma_{v}^{2}\right)}} \mathrm{e}^{-\frac{\left(\tilde{v}_{k}-\theta_{i} \tilde{s}_{k}\right)^{2}}{2\left(\theta_{i}^{2} \sigma_{s}^{2}+\sigma_{v}^{2}\right)}}+\beta g\left(\tilde{v}_{k}\right),
$$

where $\alpha_{i}>0, \beta>0, \sum_{i}^{M} \alpha_{i}+\beta=1$, and where $g(\cdot)$ is the pdf of $\tilde{v}_{k}$ given that the clutch is disengaged. The weights $\alpha_{i}$ and $\beta$ reflect the probability of the different gears and of that the clutch is engaged or disengaged, respectively. Then the log-likelihood function for $\theta$ given the measurement $\tilde{\mathbf{v}}=\left[\tilde{v}_{1}, \ldots, \tilde{v}_{N}\right]$ and $\tilde{\mathbf{s}}=\left[\tilde{s}_{1}, \ldots, \tilde{s}_{N}\right]$ can be written as

$$
\ln \mathcal{L}(\boldsymbol{\theta} \mid \tilde{\mathbf{v}}, \tilde{\mathbf{s}})=\sum_{k=1}^{N} \ln p\left(\tilde{v}_{k} \mid \tilde{s}_{k} ; \boldsymbol{\theta}\right) .
$$

Based on the $\log$-likelihood function and assuming $\tilde{\mathbf{v}}$ and $\tilde{\mathbf{s}}$ to contain a sufficient number of samples with each gear engaged, in principle the gear scale factors $\boldsymbol{\theta}$ can be estimated in an ML-sense [6]. From the constraints (5) define the set

$$
\Theta=\left\{\boldsymbol{\theta} \in \mathbb{R}^{M}: \theta_{i}<\theta_{j} \forall i<j, \theta_{1}>0\right\} .
$$

Then the ML-estimate of $\boldsymbol{\theta}$ is

$$
\hat{\boldsymbol{\theta}}_{M L}=\underset{\boldsymbol{\theta}}{\arg \max }(\ln \mathcal{L}(\boldsymbol{\theta} \mid \tilde{\mathbf{v}}, \tilde{\mathbf{s}})) \quad \text { subject to } \quad \boldsymbol{\theta} \in \Theta .
$$

Unfortunately a number of unknowns (nuisance parameters) prevent us from evaluating this expression. Also, even if all quantities but $\boldsymbol{\theta}$ where known in general the expression (8) would have to be evaluated numerically. However, the high dimensionality and the large number of samples would make a global search infeasible. The nuisance parameters can be handled by either including them in the estimation or by setting them based on heuristics. The numerical evaluation will have to be handled by some iterative non-linear optimization methods which in turn need to be initialized sufficiently close to the true value to ensure convergence. This initialization will have to be done based on heuristic methods. These heuristic methods are described in the following subsections.

\subsection{Handling nuisance parameters}

The nuisance parameters and unknowns of the estimator (8) are the noise standard deviations $\sigma_{s}$ and $\sigma_{v}$, the weights $\alpha_{i}$ and $\beta$, and the unknown distribution $g\left(\tilde{v}_{k}\right)$.

The noise standard deviations $\sigma_{s}$ and $\sigma_{v}$ are properties of the measurements and therefore it is natural to include them among the estimation parameters. This means that we would treat (6) and hence also (7) to be parameterized by $\boldsymbol{\theta}, \sigma_{s}$ and $\sigma_{v}$. Then instead of (8) the estimator would read

$$
\begin{gathered}
{\left[\hat{\boldsymbol{\theta}}_{M L}, \hat{\sigma}_{s}, \hat{\sigma}_{v}\right]=\underset{\left[\boldsymbol{\theta}, \sigma_{s}, \sigma_{v}\right]}{\arg \max }\left(\ln \mathcal{L}\left(\boldsymbol{\theta}, \sigma_{s}, \sigma_{v} \mid \mathbf{v}, \mathbf{s}\right)\right)} \\
\text { subject to } \quad \boldsymbol{\theta} \in \Theta, \sigma_{s}>0, \sigma_{v}>0 .
\end{gathered}
$$

The weighting factors $\alpha_{i}$ and $\beta$ could also possibly be included among the estimation parameters just as $\sigma_{s}$ and $\sigma_{v}$ but to limit the dimensionality of the estimation problem it would be preferable to set them based on prior knowledge. Their true value will be dependent on the driving conditions and the driver. However, if we ignore the traffic jam situation the Gaussian terms of (6) will typically have a much larger likelihood contribution than the $\beta g\left(\tilde{v}_{k}\right)$-term (the clutch will only be disengaged a small fraction of the driving time and the corresponding values in the $\left(\tilde{v}_{k}-\theta_{i} \tilde{v}_{k}\right)$-domain will be spread out) and if adjacent $\alpha_{i}$ are of the same order and there is no significant overlap between hypothesis of different engaged gears, i.e.,

$$
2 \cdot\left|\theta_{i} s_{k}-\theta_{j} s_{k}\right| \gtrsim \sqrt{\theta_{i}^{2} \sigma_{s}^{2}+\sigma_{v}^{2}} \quad \forall i \neq j, k,
$$

the contribution from a single sample in (7) from an engaged gear $\theta_{i}$ will essentially only scale with corresponding $\alpha_{i}$ value. This means 
that for good initial values the estimator will be rather insensitive to $\alpha_{i}$ value settings. If these conditions are fulfilled and large training data sets with mixed driving conditions are considered, general values for $\alpha_{i}$ and $\beta$ can be set, based on experience from typical driving behavior.

Finally the unknown distribution $g\left(\tilde{v}_{k}\right)$ has to be handled. Treating each time instant $k$ independently there is little information available about $g\left(\tilde{v}_{k}\right)$. What can be expected is $g\left(\tilde{v}_{k}\right)$ to be quite uniform with bias toward low values and with a spike at zero (vehicle stationary). Once again as with $\alpha_{i}$ and $\beta$, ignoring extreme conditions such as traffic jams and for large data sets with mixed driving conditions, a general distribution can be set by experience from typical driving behavior

\subsection{Finding initial parameter estimates}

For samples corresponding to high speeds assume $v_{k} \gg w_{k}^{v}$. Then

$$
\frac{\tilde{v}_{k}}{\tilde{s}_{k}}=\frac{v_{k}+w_{k}^{v}}{s_{k}+w_{k}^{s}} \approx \frac{v_{k}}{s_{k}+w_{k}^{s}} .
$$

Define the set,

$$
\kappa_{M}=\left\{k: \tilde{v}_{k}>d_{M} \cdot \max _{k}\left(\tilde{v}_{k}\right)\right\},
$$

where $d_{M}<1$ is a constant close to unity, containing the time instants corresponding to speed measurements close to the maximum encountered speed. For this set we can expect the top gear to be engaged, i.e.,

$$
\frac{v_{k}}{s_{k}}=\theta_{M} \quad \forall k \in \kappa_{M} .
$$

Since $w_{k}^{s}$ are assumed symmetrically distributed $\theta_{M}$ can be estimated by

$$
\hat{\theta}_{M}:=\mu_{1 / 2}\left(\left\{\tilde{v}_{k} / \tilde{s}_{k}: k \in \kappa_{M}\right\}\right),
$$

where $\mu_{1 / 2}(\cdot)$ denotes the median. This gives an initial estimate of $\theta_{M}$. The robustness of the median estimator, allowing up to $50 \%$ outliers (samples related to other gears or disengaged clutch instants), ensures that this is a reasonable estimate.

Next $\hat{\sigma}_{s}$ and $\hat{\sigma}_{v}$ need to be initialized. Due to subsequent refinement of the estimates (see (15)) it is sufficient to initialize them such that they cover a reasonable share of the data corresponding to $\kappa_{M}$. Since data corresponding to only a single scale factor $\theta_{M}$ is making contributions from $\hat{\sigma}_{s}$ and $\hat{\sigma}_{v}$ indistinguishable we assume $\sigma_{s} \approx \sigma_{v}$ and set

$$
\hat{\sigma}_{l}:=\frac{1}{\sqrt{1+\hat{\theta}_{M}^{2}}} \nu_{d_{\%}}\left(\left\{\tilde{v}_{k}-\hat{\theta}_{M} \tilde{s}_{k}: k \in \kappa_{M}\right\}\right) \forall l \in\{s, v\},
$$

where $\nu_{d_{\%}}(\cdot)$ denotes the $d_{\%}$-th percentile.

To go from here it has to be assumed that we have some knowledge about approximative ratios between different scale factors,

$$
\frac{\theta_{i+1}}{\theta_{i}} \approx c_{i} \quad \forall i \in\{1,2, \ldots, M-1\}
$$

for some constants $c_{i}>1$. This is a reasonable assumption since due to power-band and vehicle momentum considerations the gears will have typical ratios. For example the ratios for Land Rover Defender GFT MT-82 (example of wide-ratio gearbox) are less than $20 \%$ larger than those for Porsche GT3 911 G96.96 (example of close-ratio gearbox). Initial estimates of all scale factors can then be found by

$$
\hat{\theta}_{i}:=\hat{\theta}_{i+1} / c_{i} \quad \forall i \in\{1,2, \ldots, M-1\} \quad \text { given } \hat{\theta}_{M} .
$$

Unfortunately, these initial estimates are unsatisfactory since errors in $\hat{\theta}_{M}$ and in (13) will give propagating errors in $\hat{\theta}_{i}$ for decreasing values of $i$. This can be overcome by sequentially, from high to low values of $i$, refining one estimate at a time and based on this estimate update estimates for lower values of $i$. Initially also the standard deviations $\hat{\sigma}_{s}$ and $\hat{\sigma}_{v}$ need to be refined. We begin by

$$
\begin{gathered}
{\left[\hat{\theta}_{M} \hat{\sigma}_{v}, \hat{\sigma}_{s}\right]:=\underset{\hat{\theta}_{M}, \hat{\sigma}_{v}, \hat{\sigma}_{s}}{\arg \max }\left(\ln \mathcal{L}\left(\hat{\boldsymbol{\theta}}, \hat{\sigma}_{v}, \hat{\sigma}_{s} \mid \tilde{\mathbf{v}}, \tilde{\mathbf{s}}\right)\right)} \\
\text { subject to } \hat{\boldsymbol{\theta}} \in \Theta, \hat{\sigma}_{s}>0, \hat{\sigma}_{v}>0, \\
\hat{\theta}_{i-1}:=\hat{\theta}_{i} \cdot c_{i} \quad \forall i \leq M
\end{gathered}
$$

and then for consecutively lower values of $i$

$$
\begin{gathered}
\hat{\theta}_{i}:=\underset{\hat{\theta}_{i}}{\arg \max }(\ln \mathcal{L}(\hat{\boldsymbol{\theta}} \mid \tilde{\mathbf{v}}, \tilde{\mathbf{s}})) \quad \text { subject to } \quad \hat{\boldsymbol{\theta}} \in \Theta, \\
\hat{\theta}_{j-1}:=\hat{\theta}_{j} \cdot c_{j} \quad \forall j \leq i .
\end{gathered}
$$

In other words, starting with the highest scale factor and continuing with consecutively lower ones, we maximize the likelihood function with respect to a single scale factor estimate over a range (between the adjacent scale factors estimates) and update the estimate of the scale factors of lower gears.

\subsection{Final estimates}

Performing all the steps (11)-(16) the resulting estimates $\hat{\boldsymbol{\theta}}, \hat{\sigma}_{s}$ and $\hat{\sigma}_{v}$ are used as initial values for (9) which is then used to give the final scale factors estimates.

\section{MEASUREMENT CAMPAIGN}

To verify the functionality of the estimation method a measurement campaign was conducted. The vehicle used for the measurement was a BMW 520i (-96) with manual gearbox with $M=5$ gears. Speed measurements were given by $1[\mathrm{~Hz}]$ GPS doppler measurements from a GPS receiver mounted on the roof of the car and measurements by a sound-card plugged into the cigarette lighter outlet. The sampling of the signal was done with $5.1[\mathrm{kHz}]$. The measurements were recorded on a lap-top computer and processed off-line. The time synchronization was done by time stamping and manual inspection. A FFT was applied to the signal over a window length of $100[\mathrm{~ms}]$ centered around the GPS outputs. A clear frequency spike appearing in the range $50-200[\mathrm{~Hz}]$ was tracked.

Two types of measurement sets were recorded.

- 5 measurement sets of 3 minutes driving on each gear separately with the clutch engaged at all times. These sets were used to get reference estimates for the scale factors $\boldsymbol{\theta}_{i}$.

- 2 measurement sets of 20 minutes driving under mixed driving conditions. The driving contained about equal part city, residential area, highway, and country side driving.

A rough image of the mixed driving condition data is given by the histogram of $\tilde{v}_{k} / \tilde{s}_{k}$ shown in Fig. 1. The clusters are interpreted to correspond to the different gear scale factors $\theta_{i}$ and the spread out values to time instants during which the clutch is disengaged.

Mechanical references for the ratios of different scale factors were available from the technical specification of the gearbox. Their values are found in Table 1 . 


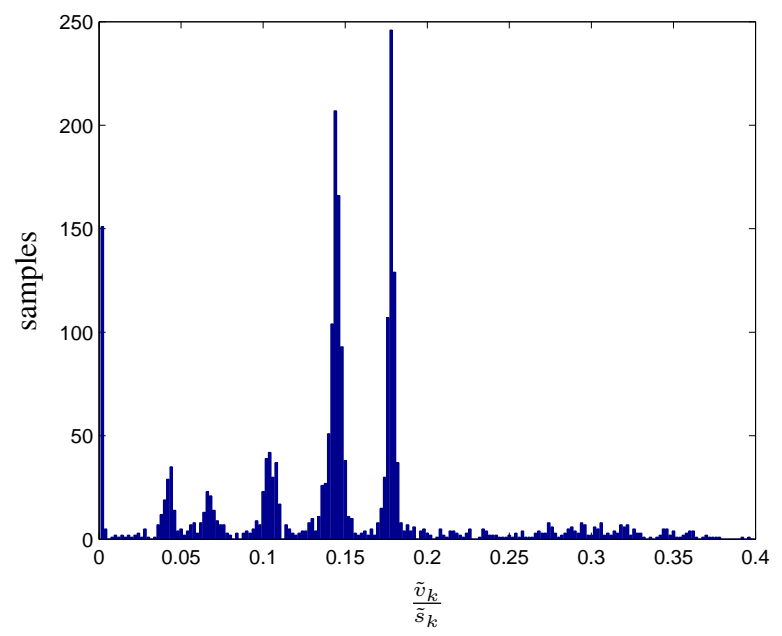

Fig. 1. Histogram (200 bins and approx. 2400 samples) of $\tilde{v}_{k} / \tilde{s}_{k}$ for 40 minutes of driving data from mixed driving conditions. The clusters are interpreted to correspond to the different gear scale factors $\left(\tilde{v}_{k} / \tilde{s}_{k} \approx \theta_{i}\right)$ and the spread out values to time instants during which the clutch is disengaged. The peak at zero corresponds to stationary time instants.

Table 1. Mechanical references (top row) from gearbox specifications and ratios of reference estimate (bottom row) based on single gear measurements sets.

\begin{tabular}{|c|c|c|c|}
\hline $\begin{array}{l}\frac{\theta_{2}}{\theta_{1}}=1.68 \\
\hat{\theta}_{2}=1.69\end{array}$ & $\begin{array}{l}\frac{\theta_{3}}{\theta_{2}}=1.51 \\
\frac{\hat{\theta}_{3}}{\hat{\theta}_{2}}=1.49\end{array}$ & $\begin{array}{l}\frac{\theta_{4}}{\theta_{3}}=1.37 \\
\frac{\hat{\theta}_{4}}{\hat{\theta}_{3}}=1.37\end{array}$ & $\begin{array}{l}\frac{\theta_{5}}{\theta_{4}}=1.22 \\
\frac{\hat{\theta}_{5}}{\hat{\theta}_{4}}=1.22\end{array}$ \\
\hline
\end{tabular}

\section{ESTIMATION RESULTS}

The scale factor references based on the 5 single gear measurement sets were estimated in an ML-sense according to (9) but with the pdf (6) replaced by a single Gaussian term,

$$
p\left(\tilde{v}_{k} \mid \tilde{s}_{k} ; \theta_{i}\right)=\frac{1}{\sqrt{2 \pi\left(\theta_{i}^{2} \sigma_{s}^{2}+\sigma_{v}^{2}\right)}} \mathrm{e}^{-\frac{\left(\tilde{v}_{k}-\theta_{i} \tilde{s}_{k}\right)^{2}}{2\left(\theta_{i}^{2} \sigma_{s}^{2}+\sigma_{v}^{2}\right)}} .
$$

That is with the ML-estimator assuming that a single gear was used and that the clutch was engaged at all times. The resulting estimates are found in Table 3. These reference estimates can be verified against the mechanical reference by inspecting their ratios. The ratios and comparison with the mechanical references are found in Table 1.

The estimators (9), (15), and (16) were implemented with Matlab $^{\circledR}$ Optimization Toolbox ${ }^{\mathrm{TM}}$ with the interior-point algorithm. A uniform pdf over the range of $\tilde{v}_{k}$ was used for $g\left(\tilde{v}_{k}\right)$. Time instants for which $\tilde{v}_{k}<2 \hat{\sigma}_{v}$ were removed. The values of the constants used in (11)-(16) can be found in Table 2. The estimated scale factors from the two measurement data sets with mixed driving conditions are found in Table 3.

\section{CONCLUSION}

A method for estimating the scale factors for each gear between speed and frequency components of readily available signals in a
Table 2. Values for constants used in the final estimation (9) and initialization (11)-(16) of the scale factor estimation.

\begin{tabular}{llll}
\hline$\alpha_{1}=0.05$ & $\alpha_{4}=0.3$ & $d_{M}=0.95$ & $c_{2}=1.35$ \\
$\alpha_{2}=0.1$ & $\alpha_{5}=0.4$ & $d_{\%}=10$ & $c_{3}=1.50$ \\
$\alpha_{3}=0.1$ & $\beta=0.1$ & $c_{1}=1.25$ & $c_{4}=1.7$ \\
\hline
\end{tabular}

Table 3. Scale factors estimates based on single gear and mixed driving conditions measurement sets, respectively.

Reference estimates - single gear measurement sets

\begin{tabular}{llll}
\hline$\theta_{1}=0.043$ & $\theta_{2}=0.072$ & $\theta_{3}=0.106$ & $\theta_{4}=0.146$
\end{tabular}

$\theta_{5}=0.178$

Estimates - mixed driving condition data set 1

$\begin{array}{llll}\theta_{1}=0.043 & \theta_{2}=0.069 & \theta_{3}=0.103 & \theta_{4}=0.145\end{array}$

$\theta_{5}=0.179$

Estimates - mixed driving condition data set 2

\begin{tabular}{llll}
\hline$\theta_{1}=0.039$ & $\theta_{2}=0.067$ & $\theta_{3}=0.106$ & $\theta_{4}=0.144$
\end{tabular}

$\theta_{5}=0.178$

motorized vehicle has been proposed. The method use training data of measurements of speed and the signal in question but no information about engaged gear or the clutch state. The method has been shown to work well for a test case in which speed and the voltage level of the electrical system were recorded on a car driving under mixed driving conditions.

\section{REFERENCES}

[1] C. Bohn, A. Cortabarria, V. Härtel, and K. Kowalczyk, "Active control of engine-induced vibrations in automotive vehicles using disturbance observer gain scheduling," Control Engineering Practice, vol. 12, pp. 1029-1039, Aug 2004.

[2] J.M. Chicharro, A.L. Morales, R. Moreno, A.J. Nieto, and P. Pintado, "Sensorless automotive engine speed measurement by noise analysis," in Proc. ICM2009, Apr 2009, Málaga, Spain.

[3] V. Cevher, R. Chellappa, and J. McClellan, "Vehicle speed estimation using acoustic wave patterns," IEEE Trans. Signal Process., vol. 57, pp. 30-47, Jan 2009.

[4] I. Skog and P. Händel, "In-car positioning and navigation technologies - a survey," IEEE Trans. Intell. Transp. Syst., vol. 10, pp. 4-21, Mar 2009.

[5] P. Händel, "Discounted least-squares gearshift detection using accelerometer data," IEEE Trans. Instrum. Meas., vol. 58, pp. 3953-3958, Dec 2009.

[6] Steven M. Kay, Fundamentals of Statistical Signal Processing, Volume 1: Estimation Theory, Prentice Hall, 1993. 